(2) Open Access Full Text Article

REVIEW

\title{
Importance of family/social support and impact on adherence to diabetic therapy
}

This article was published in the following Dove Press journal:

Diabetes, Metabolic Syndrome and Obesity:Targets and Therapy

5 November 2013

Number of times this article has been viewed

Tricia A Miller

M Robin DiMatteo

Department of Psychology, University of California, Riverside, Riverside, CA, USA
Correspondence: Tricia A Miller

Department of Psychology,

University of California, Riverside,

900 University Avenue,

Riverside, CA 92521, USA

Email trishmillerI22@gmail.com
Abstract: Diabetes mellitus affects 24 million individuals in the US. In order to manage their diabetes successfully, patients must adhere to treatment regimens that include dietary restrictions, physical activity goals, and self-monitoring of glucose levels. Numerous factors affect patients' ability to adhere properly, eg, self-efficacy, treatment expectations, health beliefs, and lack of social support. Consequently, diabetes management can be quite complex, requiring lifelong commitment and drastic changes to the patient's lifestyle. Empirical studies have shown positive and significant relationships between social support and treatment adherence among patients with diabetes. Social support from family provides patients with practical help and can buffer the stresses of living with illness. However, the exact mechanism by which social support affects patient adherence is not yet completely understood. Further research is needed to address how the differences in types of support, such as functional or emotional support, are linked to outcomes for patients. The purpose of this review is to summarize what is known of the impact of social and family support on treatment adherence in patients with diabetes and to explore the current methods and interventions used to facilitate family support for diabetic patients.

Keywords: patient adherence, patient compliance, diabetes management, support, family, social

\section{Introduction}

Diabetes mellitus affects nearly 24 million Americans, making diabetes one of the most common chronic conditions in the US. ${ }^{1}$ Current empirical research suggests that the number of individuals diagnosed with diabetes (per year) is expected to double by the year 2034. ${ }^{1}$ Diabetes is considered to be the leading cause of heart disease, stroke, kidney failure, lower limb amputation, and blindness among US adults. ${ }^{2,3}$ According to the American Diabetes Association, the management of diabetes can be costly, generating both large direct ( $\$ 116$ billion) and indirect ( $\$ 68$ billion) medical costs. ${ }^{4}$ Diabetic patients spend an average of $\$ 10,000$ per year compared with $\$ 2,700$ for nondiabetic patients; most of these costs are associated with hospitalizations due to chronic diabetes-related complications. ${ }^{4}$

The primary goals in the treatment of diabetes are for patients to maintain proper metabolic control and to reduce the risks of health complications. ${ }^{5}$ In recent correlational studies, strong associations between patient nonadherence (or noncompliance) and progression or development of diabetes-related complications have been found. ${ }^{6}$ Research by Gherman et al suggests that increases in adherence can result in patients who are more confident in self-care behaviors, and thus have better overall outcomes. ${ }^{6}$ 
The most common forms of diabetes mellitus are type 1 and type 2 . Type 1 diabetes mellitus is described as autoimmune destruction of insulin, whereas type 2 diabetes mellitus results from reduced insulin sensitivity and secretion, and is associated with obesity and hypertension. ${ }^{7}$ Adherence to treatment for type 1 and 2 diabetes mellitus includes tasks such as medication-taking, daily insulin injections, self-monitoring of glucose levels (daily or several times per day), complex dietary restrictions, frequent eye examinations, and daily exercise routines. ${ }^{1,6}$ Additionally, patients' ability to self-manage their health behavior plays a crucial role in diabetes management, given that most diabetics provide more than $90 \%$ of their own daily care. ${ }^{5}$

Patients' efforts to maintain and adhere properly to diabetes management directives often take place in social settings and can alter family and social dynamics. ${ }^{5,8,9}$ According to research by DiMatteo, support from friends and family promotes adherence by encouraging optimism and self-esteem, which can buffer the stress of being ill and reduce patient depression. ${ }^{10}$ While social support can influence the ability to adjust and live with illness, some empirical studies have reported opposite findings, such that social support can be a significant barrier to patients' self-management. ${ }^{6,9}$ The extent to which social support affects health outcomes and adherence to diabetes treatment has important implications for both policy and practice. The purposes of this review are to summarize what is known of the impact of social support on adherence to diabetes treatment and to explore the types of adherence interventions and methods used to promote social support for patients with diabetes.

\section{Scope of adherence problem}

The term nonadherence (or noncompliance) describes the extent to which patients do not follow treatment recommendations given by their health care providers. ${ }^{11}$ Nonadherence can be intentional or unintentional, where treatment advice is misunderstood, carried out incorrectly, or ignored. ${ }^{11}$ Rates of nonadherence vary widely across different disease conditions, treatment regimens, and patient populations. ${ }^{10}$ On average, $25 \%$ of patients are nonadherent to treatment. For patients with chronic disease (eg, diabetes), rates of nonadherence are $50 \%$ or more. ${ }^{11,12}$ Additionally, among children and adolescents, 50\%-70\% are nonadherent to medication directives. ${ }^{13}$ The economic burden of nonadherence due to preventable complications, hospitalizations, and poor disease outcomes is significant. However, patients and health care professionals fail to recognize that the cause of poor health outcomes may be due to patient nonadherence. ${ }^{12,14}$

Nonadherence can occur for myriad reasons, including the financial constraints associated with treatment, medication side effects, difficulty in managing complex treatment regimens, inadequate patient health literacy, and lack of social support. ${ }^{10,11,15-17}$ Significant health risks in the care of many chronic diseases are also associated with nonadherence. For example, in diabetic patients, nonadherence is associated with poor glycemic control and long-term health complications, such as retinopathy, neuropathy, and renal disease. ${ }^{5,8}$ For patients with human immunodeficiency virus, the consequence of nonadherence is viral replication and significant disease progression. ${ }^{11}$ Additionally, for patients suffering from coronary heart disease, nonadherence to medication directives can drastically jeopardize health and in some cases lead to death. ${ }^{14}$ Thus, nonadherence can compromise patient outcomes in many ways. Some research suggests that adherence to even a placebo can be beneficial to patients' health. ${ }^{14}$

Several decades of research have focused on understanding the inherent factors leading to change in adherence behaviors. One such factor is the effect of social support on patient adherence to diabetes management. ${ }^{18}$ Previous research by Glasgow and Toobert found that family support was the strongest and most consistent predictor of adherence to treatment in patients with type 2 diabetes. ${ }^{19}$ In another study of women with gestational diabetes, adherence to dietary recommendations was strongly associated with familial social support. ${ }^{18}$ More recently, among children with type 1 diabetes, family conflict, perceptions of nonsupportive parents, and lack of responsibility for diabetes management were associated with poor patient adherence. ${ }^{5,18}$ In contrast, positive dimensions of family functioning, including family guidance and control over the management of diabetes, were related to increases in adherence behaviors. ${ }^{5,18}$ Furthermore, among elderly patients, social support from family and friends helps patients to remain active in their care when faced with physical, social, and economic vulnerabilities. ${ }^{10,20}$

Research by DiMatteo suggests that the relationship between social support and health outcomes may be mediated by patient adherence. ${ }^{10}$ Further investigation is needed to identify the specific types of social support (ie, structural, functional, practical, and emotional) that are most effective in enhancing various diabetes management activities. The unique ways in which family members can provide support for patients with diabetes also deserve investigation. In the 
following section, aspects of the family and social supportadherence relationship will be discussed.

\section{Impact of family support on adherence}

The association between disease management and social support has been researched extensively in the social and behavioral sciences. The precise mechanism by which social support contributes to health outcomes is not yet completely understood. Research does suggest, however, that social support can benefit patients' health by buffering stress, changing affective states, increasing self-efficacy, and influencing change in negative health behaviors. ${ }^{10}$ Rosland et al found that practical and emotional support received by both family and friends had a positive influence on global measures of disease management in patients with diabetes. ${ }^{9}$ In fact, a meta-analytic review of 122 empirical studies found that adherence was $27 \%$ higher when patients had practical support available to them. ${ }^{10}$ Additionally, research by Pereira et al indicates strong associations between positive family dimensions (eg, cohesion and familial guidance) and better glycemic control among diabetic patients. ${ }^{21,22} \mathrm{In}$ regards to family cohesion, in which families are described as warm, accepting, and close, the odds of adherence were three times higher when compared with noncohesive families. ${ }^{10}$ Furthermore, family structural support (ie, patient's marital status and living arrangement) is also positively associated with treatment adherence. In research by DiMatteo, the odds of adherence for married patients were 1.27 times higher than for unmarried patients. ${ }^{10}$ Additionally, "living with others" had positive effects on adherence for adults $(r=0.08$, $P<0.05) .{ }^{10}$ More specifically, the effect of patient adherence and "living with others" was stronger for behavioral regimens compared with medication regimens. ${ }^{10}$ Moreover, in the same study, functional social support (eg, practical and emotional support) had stronger effects on adherence than did structural social support, suggesting that the quality of family-patient relationships matters more than just the mere presence of individuals within a patient's network of support. ${ }^{10}$

Social support has also been linked to negative health outcomes. ${ }^{9}$ Some studies suggest that patients often feel criticized or nagged, and sometimes even guilt, when receiving support from family. ${ }^{23}$ Other studies have shown competing demands between patient and family members as barriers to self-management. ${ }^{24}$ For example, family members may not want to eat the same foods as a diabetic patient who is trying to maintain a healthier diet. Such competing demands limit patients' time and energy and introduce stress that can negatively affect patients who are trying to juggle multiple family roles while living with illness (ie, parents or spouse). ${ }^{24}$

Among adolescents, increases in responsibility for disease management have been linked to higher levels of parentadolescent conflict and nonadherence to treatment. In a study of patients with cystic fibrosis, adolescents were responsible for almost $90 \%$ of their own daily treatment, resulting in significantly lower rates of adherence than if parents were involved. ${ }^{25}$ Parent-adolescent conflicts stem from differences in perceptions and expectations of autonomy; these discrepancies in decision-making autonomy are referred to as DDMA. ${ }^{26}$ Studies involving adolescents with type 1 diabetes have found significant and positive associations between adolescent-reported family support, improved treatment adherence, and decreases in DDMA. ${ }^{8}$ In order to manage diabetes effectively, adolescents, parents, and health professionals must mutually agree upon a family-based treatment plan that accurately reflects the abilities of the adolescent. ${ }^{27}$ Thus, the ways in which social support directly and indirectly affects outcomes in adult and adolescent patients can be quite complicated. Current research points to the importance of designing interventions to improve adherence that include practical help, emotional support, and cohesive networks in the context of diabetes care. ${ }^{10}$

\section{Methods to build positive family support}

Most theories of health and behavior change suggest a need for social support as a crucial component; family members are the most significant source of that support. ${ }^{28,29}$ Even with correlational evidence suggesting the importance of social support, few intervention studies have targeted familial support as a means of diabetes management in adults. ${ }^{29} \mathrm{In}$ the studies that have included family support, results are promising although somewhat inconsistent. ${ }^{29}$ For example, in a study conducted by Robinson, a diabetes education and family social support training program was used to assist elderly diabetic patients who had difficulty adhering to dietary restrictions..$^{30}$ The training program had five goals: to educate patients about the development of diabetes and how to manage living with illness; to allow patients to discuss thoughts and feelings and accompanying lifestyle changes associated with diabetes; to facilitate self-esteem and help patients cope and take control of their illness; to develop solutions and techniques to maintain proper diet; and to 
help patients develop supportive relationships among family members to maintain dietary adherence over time. ${ }^{30}$ The results of this study revealed that patients who participated in the training program had reduced blood glucose levels and increases in adherence for up to 24 weeks after completion of the training program. ${ }^{30}$ In another study, Wing et al compared an individual weight loss intervention with a weight loss intervention that encompassed the support of a spouse and found no significant difference in weight loss, calorie intake, and glycemic control between groups. ${ }^{31}$ In Gilliland et al, researchers looked at differences across psychoeducational groups among adults and their families, psychoeducational groups without patients' families, and a control group. ${ }^{32}$ Results from this study revealed only small increases in glycemic control between the intervention groups and the control group. ${ }^{32}$ Mayberry et al conducted focus groups to explore the relationship between family support and adherence to medication regimens for adults with diabetes. ${ }^{29}$ Their results revealed that instrumental support (or observable actions that help patients manage their illness) was the most common type of social support. ${ }^{29}$ Patient-reported examples of instrumental support included tasks such as spouses maintaining medical appointments and doing the grocery shopping. ${ }^{29}$ Additionally, some patients in the focus group expressed frustration with family members "sabotaging" or offering "miscarried helping" behaviors. ${ }^{29}$ These findings suggest that increases in family involvement can create conflict and potentially even undermine patients' success at performing diabetes self-care tasks. ${ }^{29}$

Furthermore, the empirical literature on social support and the management of diabetes among adolescents and their families has revealed significant and positive effects on patient adherence to treatment. In a randomized controlled trial, adolescents with type 1 diabetes and their families participated in ten sessions of behavioral family systems therapy. Behavioral family systems therapy is a familyfocused multifaceted intervention that targets communication between family members, problem-solving skills, family beliefs that affect communication, and potential barriers to problem-solving tasks. ${ }^{33}$ The results of this study yielded significant improvements in parent-adolescent relationships and family communication as reported by parents and adolescents. ${ }^{33}$ However, behavioral family systems therapy had no impact on glycemic control or adherence to treatment.

Interestingly, Wysocki et al sought to determine whether a revised version of behavioral family systems therapy specific to diabetes (BFST-D) would significantly improve adherence. ${ }^{34}$ In this revision, BFST-D consisted of problemsolving training, communication training, cognitive restructuring, and functional-structural family therapy. ${ }^{34,35}$ As predicted, BFST-D significantly improved family conflict, metabolic control, and adherence to treatment among families of adolescents with poor metabolic control at baseline. ${ }^{34,35}$

Additionally, peer support and problem-solving group interventions have been shown to improve health outcomes for adolescents with type 1 diabetes. In a study by Løding et al, adolescents and their parents participated in peer discussions where they were given the opportunity to focus on education and problem-solving strategies to encourage adolescents to take more responsibility in diabetes management. ${ }^{36}$ In doing so, adolescents showed increases in satisfaction and the ability to communicate more calmly with their parents. ${ }^{36}$ Adolescents also reported fewer objections to measuring their glucose values and injecting insulin in public after the intervention. ${ }^{36}$

\section{Future research and practice}

Research concerning the effects of social support on patient adherence to diabetes management suggests important implications for future research and clinical practice. First, with the exceedingly large proportion of patients who are nonadherent, intervention studies should focus on different types of social support that may lead to significant increases in adherence behaviors. For example, in Van der Wulp et al, researchers examined the effectiveness of a peer-led self-management intervention in patients recently diagnosed with type 2 diabetes. ${ }^{37}$ Patients in the intervention group received visits from an experienced peer (someone with diabetes who is adherent to treatment and lifestyle guidelines), and together patients and peers set goals and formulated plans to maintain self-care behaviors over time. ${ }^{37}$ Patients who participated in the intervention experienced improvements in self-efficacy, coping, and saturated fat intake. ${ }^{37}$ More recently, integrative health coaching support for patients with type 2 diabetes has been associated with increases in positive health outcomes. Integrative health coaching is a personalized style of intervention that allows patients to identify their own values and expectations for their health. ${ }^{38}$ Wolever et al found that patient activation and perceived social support increased as a result of integrative health coaching. ${ }^{38}$

Efforts to improve adherence and social support in patients with diabetes have also been explored through technology-based interventions. ${ }^{39}$ In Arora et al, researchers developed a mobile health text message-based intervention 
(ie, mHealth) targeted at improving adherence and self-efficacy in low-income diabetic patients. ${ }^{39}$ Patients who enrolled in the TExT-MED program received three daily text messages over the course of 3 weeks. ${ }^{39}$ Each message delivered was based on the following domains: education/ motivation, medication reminders, healthy living challenges, diabetes trivia, and links to diabetes management resources and tools. ${ }^{39}$ Results from this study indicated significant increases in patient adherence behaviors regarding diet and exercise regimens and improved self-efficacy up to 3 weeks after the intervention. ${ }^{39}$

Moreover, in McKay et al, an Internet-based intervention known as The Diabetes Network (D-Net) was used to assess the effects of peer support on self-management behaviors. ${ }^{40}$ The purpose of D-Net was to provide peer support and personalized self-management and dietary feedback via the Internet. Each patient was provided with a D-Net computer with which they could access diabetes education, interact with a dietary management coach, and participate in a peerdirected forum in which patients could discuss difficulties in coping with other diabetic patients. ${ }^{40}$ As a result of the intervention, improvements in adherence to dietary recommendations were found. ${ }^{40}$ Furthermore, in the clinical setting, research suggests that the quality and types of patients' relationships are instrumental in assisting patients to receive the most benefit from their treatments. ${ }^{10}$ Thus, the conducting of more evidence-based interventions examining the quality and type of relationships that affect patient adherence should be a clinical priority. ${ }^{10}$

\section{Conclusion}

Family and social support are important aspects of adherence to diabetes management. Numerous correlational studies have shown a positive and significant relationship between social support and adherence to diabetes treatment. Future research should identify the many types of social support interventions that promote adherence; in doing so, diabetic patients are given the ability to seek social support that is most conducive and appropriate for their lifestyle. Lastly, further empirical evidence is needed to address the mechanisms by which social support works to directly influence health outcomes, health care utilization, and behavior change.

\section{Acknowledgment}

The authors would like to thank the following research assistants for helping with this review: Allen Roughton, Shiromi Ratnayake, and Amberly Navalta.

\section{Disclosure}

The authors report no conflict of interest in this work.

\section{References}

1. Bailey G, Barner J, Weems J, et al. Assessing barriers to medication adherence in underserved patients with diabetes in Texas. Diabetes Educ. 2012;38:271-279.

2. Centers for Disease Control and Prevention. Diabetes public health resource. Available from: http://www.cdc.gov/diabetes/. Accessed September 18, 2013

3. Egede L, Gebregziabher M, Dismuke C. Medication nonadherence in diabetes longitudinal effects on costs and potential cost savings from improvement. Diabetes Care. 2012;35:2533-2539.

4. American Diabetes Association. Economic costs of diabetes in the US in 2007. Diabetes Care. 2008;31:596-615.

5. Rintala TM, Jaatinen P, Paavilainen E, Astedt-Kurki P. Interrelation between adult persons with diabetes and their family: a systematic review of the literature. J Fam Nurs. 2013;19:3-21.

6. Gherman A, Schnur J, Montgomery G, Sassu R, Veresiu I, David D How are adherent people more likely to think? A meta-analysis of health beliefs and diabetes self-care. Diabetes Educ. 2011;37:392-408.

7. American Diabetes Association. Standards of medical care in diabetes - 2013. Diabetes Care. 2013;36:S11-S66.

8. Lewandowski A, Drotar D. The relationship between parent-reported social support and adherence to medical treatment in families of adolescents with type 1 diabetes. J Pediatr Psychol. 2007;32:427-436.

9. Rosland AM, Kieffer E, Israel B, et al. When is social support important? The association of family support and professional support with specific diabetes self-management behaviors. J Gen Intern Med. 2008;23:1992-1999

10. DiMatteo MR. Social support and patient adherence to medical treatment: a meta-analysis. Health Psychol. 2004;23:207-218.

11. DiMatteo MR. Variations in patients' adherence to medical recommendations: a quantitative review of 50 years of research. Med Care. 2004;42:200-209.

12. DiMatteo MR, Miller TA. Treatment adherence in adolescence. In: O'Donohue WT, Benuto LT, Tolle LW, editors. Handbook of Adolescent Health Psychology. New York, NY: Springer; 2013.

13. Chappuy H, Treluyer JM, Faesch S, Giraud C, Cheron CG. Length of the treatment and number of doses per day as major determinants of child adherence to acute treatment. Acta Paediatr. 2009;99:433-437.

14. DiMatteo MR, Haskard-Zolnierek KB, Martin LR. Improving patient adherence: a three-factor model to guide practice. Health Psychol Rev. 2012;6:74-91.

15. Aljasem LI, Peyrot M, Wissow L, Rubin RR. The impact of barriers and self-efficacy on self-care behaviors in type 2 diabetes. Diabetes Educ. 2001;27:393-404.

16. Fortmann AL, Gallo LC, Philis-Tsimikas A. Glycemic control among Latinos with type 2 diabetes: the role of social-environmental support resources. Health Psychol. 2011;30:251-258.

17. Cohen J, Christensen K, Feldmen L. Disease management and medication compliance. Popul Health Manag. 2012;15:20-28.

18. Nicklett E, Liang J. Diabetes-related support, regimen adherence, and health decline among older adults. J Gerontol B Psychol Sci Soc Sci. 2009;65B:390-399.

19. Glasgow RE, Toobert DJ. Social environment and regimen adherence among type II diabetes patients. Diabetes Care. 1988;11:377-386.

20. Zhang X, Norris S, Gregg EW, Beckles G. Social support and mortality among older persons with diabetes. Diabetes Educ. 2007;33: 273-281.

21. Pereira M, Berg-Cross L, Almeida P, Machado J. Impact of family environment and support on adherence, metabolic control, and quality of life in adolescents with diabetes. Int J Behav Med. 2008;15:187-193.

22. Cox DJ, Gonder-Frederick L. Major developments in behavioral diabetes research. J Consult Clin Psychol. 1992;60:628-638. 
23. Carter-Edwards L, Skelly AH, Cagle CS, Appel SJ. They care but don't understand: family support of African American women with type 2 diabetes. Diabetes Educ. 2004;30:493-501.

24. Gallant M. Help or hindrance? How family and friends influence chronic illness self-management among older adults (report). Res Aging. 2007;29:375.

25. Modi AC, Marciel KK, Slater SK, Drotar D, Quittner AL. The influence of parental supervision on medical adherence in adolescents with cystic fibrosis: developmental shifts from early to late adolescents. Child Health Care. 2008;37:78-92.

26. Holmbeck GN. A model of family relational transformations during the transition to adolescence: parent adolescent conflict and adaptation. In: Graber JA, Brooks-Gunn J, Peterson A, editors. Transitions Through Adolescence: Interpersonal Domains and Context. Hillsdale, NJ: Lawrence Erlbaum Associates Inc; 1996.

27. Miller VA, Drotar D. Decision-making competence and adherence to treatment in adolescents with diabetes. J Pediatr Psychol. 2007;32: $178-188$.

28. Kang CM, Chang SC, Chen PL, et al. Comparison of family partnership intervention care vs conventional care in adults with poorly controlled type 2 diabetes in a community hospital: a randomized controlled trial. Int J Nurs Stud. 2010;47:1363-1373.

29. Mayberry LS, Osborn CY. Family support, medication adherence and glycemic control among adults with type 2 diabetes. Diabetes Care. 2012;35:1239-1245.

30. Robison F. A training and support group for elderly diabetics: description and evaluation. JSGW. 1993;18:127-136.

31. Wing RR, Marcus MD, Epstein LH, Jawad AA. A "family-based" approach to the treatment of obese type II diabetic patients. J Consult Clin Psychol. 1991;59:156-162.
32. Gillard SS, Azen SP, Perez GE, Carter JS. Strong in the body and spirit: lifestyle intervention for native American adults with diabetes in New Mexico. Diabetes Care. 2002;25:78-83.

33. Wysocki T, Harris MA, Greco P, et al. Randomized controlled trial of behavioral therapy for families of adolescents with insulin dependent diabetes mellitus. J Pediatr Psychol. 2000;25:23-33.

34. Wysocki T, Harris MA, Buckloh LM, et al. Effects of behavioral family systems therapy for diabetes on adolescents' family relationships, treatment adherence and metabolic control. J Pediatr Psychol. 2006;31:928-938.

35. Wysocki T, Harris M, Buckloh L, et al. Randomized, controlled trial of behavioral family systems therapy for diabetes: maintenance and generalization of effects on parent-adolescent communication. Behav Ther. 2008;39:33-46.

36. Løding RN, Wold JE, Skavhaug A, Graue M. Evaluation of peer-group support and problem-solving training in the treatment of adolescents with type 1 diabetes. European Diabetes Nursing. 2007;4:28-33.

37. Van der Wulp I, de Leeuw J, Gorter K, Rutten G. Effectiveness of peer-led self-management coaching for patients recently diagnosed with type 2 diabetes mellitus in primary care: a randomized controlled trial. Diabet Med. 2012;29:e390-e397.

38. Wolever R, Dreusicke M, Fikkan J, et al. Integrative health coaching for patients with type 2 diabetes: a randomized clinical trial. Diabetes Educ. 2010;36:629-639.

39. Arora S, Peters A, Agy C, Menchine M. A mobile health intervention for inner city patients with poorly controlled diabetes: proof-of-concept of the TExT-MED program. Diabetes Technol Ther. 2012;14:492-496.

40. McKay GH, Glasgow RE, Feil EG, Boles SM, Barrera M. Internetbased diabetes self-management and support: initial outcomes from the diabetes network project. Rehabil Psychol. 2002;47:31-48.

\section{Publish your work in this journal}

Diabetes, Metabolic Syndrome and Obesity: Targets and Therapy is an international, peer-reviewed open-access journal committed to the rapid publication of the latest laboratory and clinical findings in the fields of diabetes, metabolic syndrome and obesity research. Original research, review, case reports, hypothesis formation, expert opinion and commentaries are all considered for publication. The manuscript management system is completely online and includes a very quick and fair peer-review system, which is all easy to use. Visit http://www.dovepress.com/testimonials.php to read real quotes from published authors. 